\title{
Tactile Stimulation Evokes Long-Term Synaptic Plasticity in the Granular Layer of Cerebellum
}

\author{
Leda Roggeri, Bruno Rivieccio, Paola Rossi, and Egidio D'Angelo \\ Department of Cellular/Molecular Physiological and Pharmacological Sciences, University of Pavia and Consorzio Nazionale Interuniversitario per le \\ Scienze Fisiche della Materia, I-27100 Pavia, Italy
}

Several forms of long-term synaptic plasticity [long-term potentiation (LTP) and long-term depression (LTD)] have been reported in the cerebellar circuit in vitro, but their determination in vivo was still lacking in most cases. Here we show that, in the urethane-anesthetized rat, appropriate patterns of facial tactile stimulation as well as intracerebellar electrical stimulation can induce LTP and LTD in local field potentials recorded from the granular layer of Crus-IIa. LTD prevailed in control conditions, whereas LTP prevailed during local application of gabazine. No relevant plasticity was observed when gabazine and APV were coapplied. The pharmacological and kinetic properties of LTP and LTD in vivo were compatible with those reported in the granule cell layer in vitro (Mapelli and D'Angelo, 2007), suggesting that NMDA receptor-dependent plasticity was generated at the mossy fiber-granule cell synapse under the inhibitory control of the Golgi cell circuit. Interestingly, LTP and LTD were able to regulate the response latency to tactile stimulation, as expected from computational modeling of the expression mechanisms (Nieus et al., 2006). This result suggests that LTP and LTD could regulate the spatiotemporal pattern of granular layer responses to mossy fiber inputs.

Key words: LTP; LTD; cerebellum; granular layer; in vivo; tactile stimulation

\section{Introduction}

Long-term synaptic plasticity is a modification of synaptic strength, in the form of potentiation (LTP) or depression (LTD), thought to be the basis of learning and memory in the brain (Bliss and Collingridge, 1993; Malenka and Nicoll, 1999; Bliss et al., 2003). In the cerebellum, the most renowned form of plasticity is LTD at the parallel fiber-Purkinje cell synapse (Ito et al., 1982), which has been proposed to provide a mechanism for motor learning and control (Marr, 1969; Ito, 1984) [but see De Schutter (1995) and Welsh et al. (2005)]. Recently, using acute slice preparations, LTP was also shown to occur at the parallel fiber-Purkinje cell synapse. Moreover, various forms of long-term synaptic plasticity have been reported in the cerebellar circuit, including the granular layer, the molecular layer, and the deep cerebellar nuclei [for review, see Hansel et al. (2001), Ito (2006), and Jörntell and Hansel (2006)]. However, for most of them, any demonstration in vivo was still lacking.

The mossy fiber-granule cell relay of cerebellum generates NMDA receptor-dependent synaptic plasticity in acute cerebellar slices (D’Angelo et al., 1999; Rossi et al., 2002). This consists of either LTP or LTD, depending on the local excitatory/inhibitory balance: repeated high-frequency mossy fiber bursts (thetaburst) induce LTP when synaptic inhibition is weak, whereas the

\footnotetext{
Received Dec. 24, 2007; revised April 15, 2008; accepted May 12, 2008.

This work was supported by European Community Grants CEREBELLUM OLG3-CT-2001-02256 and SENSOPAC FP6-IST028056 and by Fondazione CARIPL0 of Italy. We thank Alfredo Fontanini and Arianna Maffei for participating in early parts of the investigation and Gerardo Biella and Daniela Necchi for contributing to histological analysis.

Correspondence should be addressed to Egidio D'Angelo, Physiological and Pharmacological Sciences, University

of Pavia, Via Forlanini 6, Pavia, I-27100, Italy. E-mail: dangelo@unipv.it.

DOI:10.1523/JNEUROSC1.5709-07.2008

Copyright $\odot 2008$ Society for Neuroscience $\quad 0270-6474 / 08 / 286354-06 \$ 15.00 / 0$
}

same patterns induce LTD when synaptic inhibition is strong (Mapelli and D'Angelo, 2007). The most probable explanation is that Golgi cell inhibition, by controlling granule cell depolarization, regulates NMDA channel unblock and $\mathrm{Ca}^{2+}$ influx during the bursts, configuring a BCM (Bienenstock-Cooper-Munro)like plasticity rule (Gall et al., 2005). Mossy fiber-granule cell LTP and LTD involve changes in release probability (Sola et al., 2004) (A. D’Errico, F. Prestori, P. Rossi, and E. D’Angelo, unpublished results) and intrinsic excitability (Armano et al., 2000). The combination of these mechanisms, by regulating short-term synaptic dynamics and EPSP-spike coupling, determines the delay and discharge frequency of granule cell responses to mossy fiber bursts (Nieus et al., 2006).

We report here that LTP and LTD can be induced both by intracerebellar electrical stimulation and by patterned tactile stimulation of the whisker pad in the anesthetized rat. LTP and LTD in vivo showed kinetics and induction properties resembling those previously reported in vitro for mossy fiber-granule cell synaptic plasticity and could regulate granular layer response delay. These results provide the first demonstration that LTP and LTD can be induced in the granular layer of cerebellum in vivo and suggest their involvement in cerebellar adaptation to native mossy fiber inputs.

\section{Materials and Methods}

Extracellular field recordings were performed from the granular layer of Crus-IIa in 20- to 26-d-old Wistar rats. Although patch-clamp recordings have also been obtained from cerebellar neurons in vivo (Chadderton et al., 2004; Jörntell and Ekerot, 2006), field recordings ensured the long duration $(\geq 1 \mathrm{~h}$ ) required for investigating long-term synaptic plasticity. Synaptic plasticity is indeed detectable with extracellular record- 


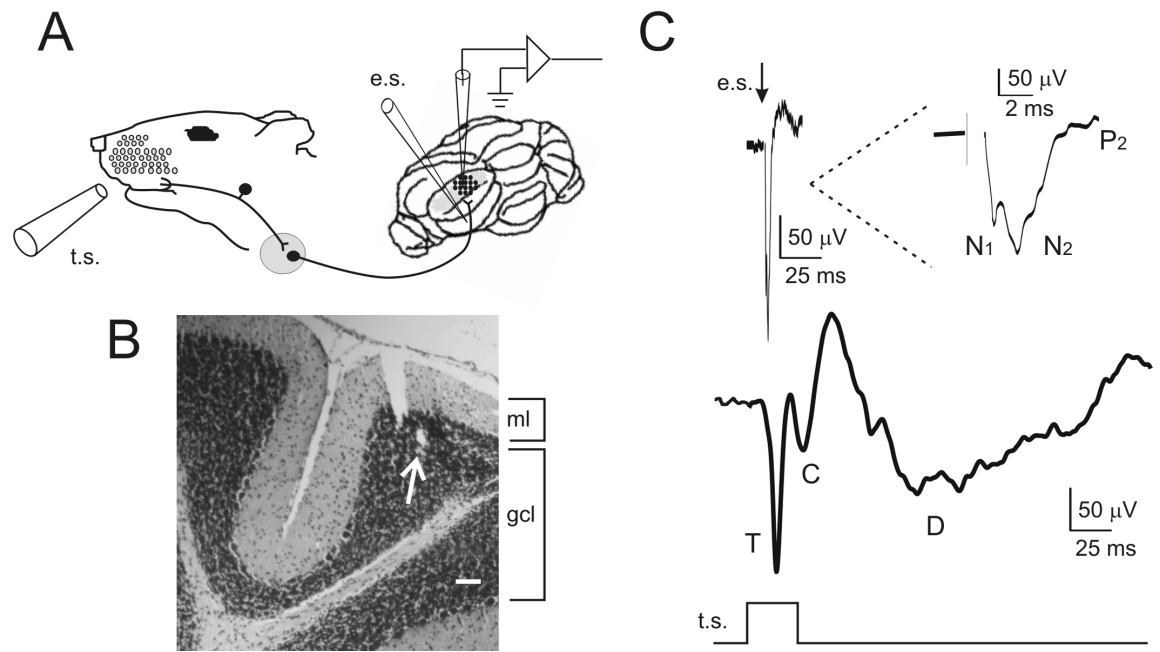

Figure 1. Local field potentials recorded from the granular layer of cerebellar Crus-lla. A, Schematic representation of LFP recordings performed from the granular layer of Crus-lla in the anesthetized rat. Tactile stimulation (t.s.) was performed by delivering air puffs to the whisker pad. Electrical stimulation of the afferent mossy fiber tract (e.s.) was performed with an intracerebellar tungsten electrode. B, A 10- $\mu \mathrm{m}$-thick histological section stained with toluidine blue. Scale bar, $100 \mu \mathrm{m}$. The arrow indicates the electrolytic lesion performed at the end of the experiment to verify the localization of the recording electrode. $\mathrm{ml}$, Molecular layer; $\mathrm{gcl}$, granule cell layer. $\boldsymbol{C}$, The traces show the LFP obtained from the granular layer using either electrical or tactile stimulation (averages of 60 responses during $10 \mathrm{~min}$ control recordings). The response to electrical stimulation shows the typical $\mathrm{N}_{1}-\mathrm{N}_{2}-\mathrm{P}_{2}$ complex generated by granular layer activation (the wave is shown enlarged in the inset). The response to tactile stimulation consists of a sequence of three waves, $T, C$, and D. Note that the $N_{1}-N_{2}-P_{2}$ complex is much shorter than the T-C-D complex.

ings in vitro, showing properties similar to those revealed with patchclamp recordings (Mapelli and D'Angelo, 2007).

For anesthesia, urethane was preferred to ketamine, because ketamine largely exerts its action by blocking NMDA receptors (up to $80 \%$ at anesthetic concentrations) (Franks and Lieb, 1994; Friedberg et al., 1999; Hara and Harris, 2002), the main receptors responsible for LTP and LTD induction in the granular layer. NMDA receptors are also critical for repetitive signal transmission along the mossy fiber pathway (Kinney and Slater, 1992), which is indeed depressed by the ketamine-xylazine mixture in the decerebrated rat (Bengtsson and Jörntell, 2007). Urethane has been reported to exert its anesthetic action through multiple weak effects, including a $10 \%$ reduction of NMDA, an $18 \%$ reduction of AMPA, and a $23 \%$ enhancement of $\mathrm{GABA}_{\mathrm{A}}$ receptor-mediated currents (Hara and Harris, 2002).

Anesthesia and surgical procedures. The rats were anesthetized with intraperitoneal injection of urethane (Sigma) dissolved in $0.9 \% \mathrm{NaCl}$ Induction $(1.35 \mathrm{~g} / \mathrm{kg})$ was followed $30 \mathrm{~min}$ later by a reinforcement $(10 \%$ of induction dose) stabilizing deep anesthesia. The level of anesthesia was assessed through withdrawal of the leg after pinching, eyeblink after an air puff, and spontaneous whisking. According to Friedberg et al. (1999), anesthesia was between Guedel stages III- 2 and III-3 and remained stable along the experiments (Friedberg et al., 1999). Electrocardiograms and electromyograms were used to monitor the heart rate $(360-420 / \mathrm{min})$ and respiratory rate $(100-120 / \mathrm{min})$, which also remained stable throughout the experiments. The animal was fixed on a stereotaxic table (Kopf 1730 equipped with custom-made ear and bite bars) covered with a heating pad. The rat body temperature, which was monitored with a rectal probe connected through a feedback temperature controller (F.S.T.) to the heating pad, was maintained at $37^{\circ} \mathrm{C}$.

The exposure of the cerebellar surface was performed following surgical procedures reported previously (Bower and Woolston, 1983; Morissette and Bower, 1996; Lu et al., 2005). Briefly, after the skin and muscles were dislocated (during this phase, cutaneous reflexes were reduced with $0.2 \mathrm{ml}$ of lidocaine; AstraZeneca) and the bone was cleaned, a $0.5 \mathrm{~mm}$ hole was drilled $2-3 \mathrm{~mm}$ posterior and 3-4 $\mathrm{mm}$ lateral from lambda on the left hemisphere to expose the cerebellar surface in correspondence to Crus-IIa. The dura mater was carefully removed, and the brain surface was dipped with standard extracellular Krebs' solution prewarmed at $37^{\circ} \mathrm{C}$. Krebs' solution contained the following (in $\mathrm{mm}$ ): $\mathrm{NaCl}$ (120), $\mathrm{KCl}(2), \mathrm{MgSO}_{4}$ (1.2), $\mathrm{NaHCO}_{3}(26), \mathrm{KH}_{2} \mathrm{PO}_{4}(1.18), \mathrm{CaCl}_{2}(2)$, and glucose (11) and was oxygenated maintaining $\mathrm{pH}$ 7.4.

LFP recordings, stimulation, drug application, and histology. Local field potentials (LFPs) from the granular layer were typically obtained at a depth of 400-600 $\mu \mathrm{m}$ with glass (borosilicate) pipettes $(3-5 \mu \mathrm{m})$ filled with $2 \mathrm{M} \mathrm{NaCl}(0.8-1$ $\mathrm{M} \Omega$ ) inserted perpendicular to the surface. The choice of small-tipped glass electrodes was aimed at increasing the spatial selectivity of recordings, because granular layer excitation is known to occur in "spots" (Shambes et al., 1978), and LTP and LTD are known to alternate over distances of $<50 \mu \mathrm{m}$ (Mapelli and D'Angelo, 2007) (D. Gandolfi, J. Mapelli, and E. D'Angelo, unpublished results). The recordings revealed indeed a high receptive-field sensitivity, indicating that signals were generated by subsets of granule cells (data not shown). Extracellular currents were recorded with an Axopatch-1D amplifier (Molecular Devices) in the voltage-clamp mode and converted to potentials dividing by the electrode resistance. The signals were bandpass filtered between $100 \mathrm{~Hz}$ (high-pass) and $2 \mathrm{kHz}$ (low-pass), digitized at $50 \mu \mathrm{s} /$ point through a Digidata 1200 interface (Molecular Devices), and stored on a PC using Clampex 8 (Molecular Devices). The same board and software were used to monitor and record body temperature and heart and respiratory rate and to generate stimulation pulses.

Electrical stimulation (70 $\mu \mathrm{s}, 5-10 \mathrm{~V}$ pulses) was performed using a coaxial platinum electrode (Micro Probe) connected to an analog pulse generator through an isolation unit. The electrode was placed $\sim 200-400$ $\mu \mathrm{m}$ below the recording electrode in Crus-IIa, respecting the geometry usually adopted for acute slice stimulation-recording experiments. Sensory stimulation was performed through a plastic $0.5 \mathrm{~mm}$ pipette connected with an MPPI-2 Pressure Injector (Applied Scientific Instrumentation) and a nitrogen cylinder. The tip was positioned $2-3 \mathrm{~mm}$ from the snout and delivered $30 \mathrm{~ms}$ pulses at $30 \mathrm{psi}$. The fact that $\mathrm{T}$ peaked with a delay slightly longer than reported in earlier experiments [13 ms vs $10 \mathrm{~ms}$ in Morissette and Bower (1996)] may reflect the weaker and smoother stimulation caused by our air puff. Once a signal was detected, the tip was moved around the perioral region to optimize the shape and stabilize the response. Control stimuli were delivered at $0.1 \mathrm{~Hz}$, and theta frequency stimulus patterns were generated to induce plasticity (see Results).

Drugs were applied directly onto the cerebellar surface at a relatively high concentration [500 $\mu \mathrm{M}$ APV and $500 \mu \mathrm{M}$ gabazine (SR95531); Tocris Cookson], as previously reported in this same preparation by Chadderton et al. (2004). Consistent effects of the drugs appeared in $<5 \mathrm{~min}$.

To confirm the location of the recording electrode, in some experiments $(n=6)$ an electrolytic lesion was performed by injecting a $10 \mathrm{~s}, 8$ $\mathrm{nA}$ current. Then, the rat was perfused transcardially with saline followed by $4 \%$ formaldehyde. The fixated brain was removed, and $70-\mu \mathrm{m}$-thick histological sections were prepared and stained with toluidine blue.

Data analysis. The extracellular signals were acquired and processed off-line with pClamp software (Molecular Devices). The signal-to-noise ratio was improved by averaging 20 consecutive traces and by digital filtering (Gaussian low-pass filter at $1 \mathrm{kHz}$ and an eight-pole Bessel highpass filter at $10 \mathrm{~Hz}$ ). Waveform peak amplitudes were measured relative to the preceding $10 \mathrm{~ms}$ baseline, and delays were measured between stimulus onset and the waveform peak. Data are reported as mean \pm SEM, and the statistical significance of results was assessed. In casecontrol measurements and for comparison between groups, the Student's $t$ test was used. On the datasets obtained using different induction protocols, the hypothesis that results were significantly different among the pharmaco- 

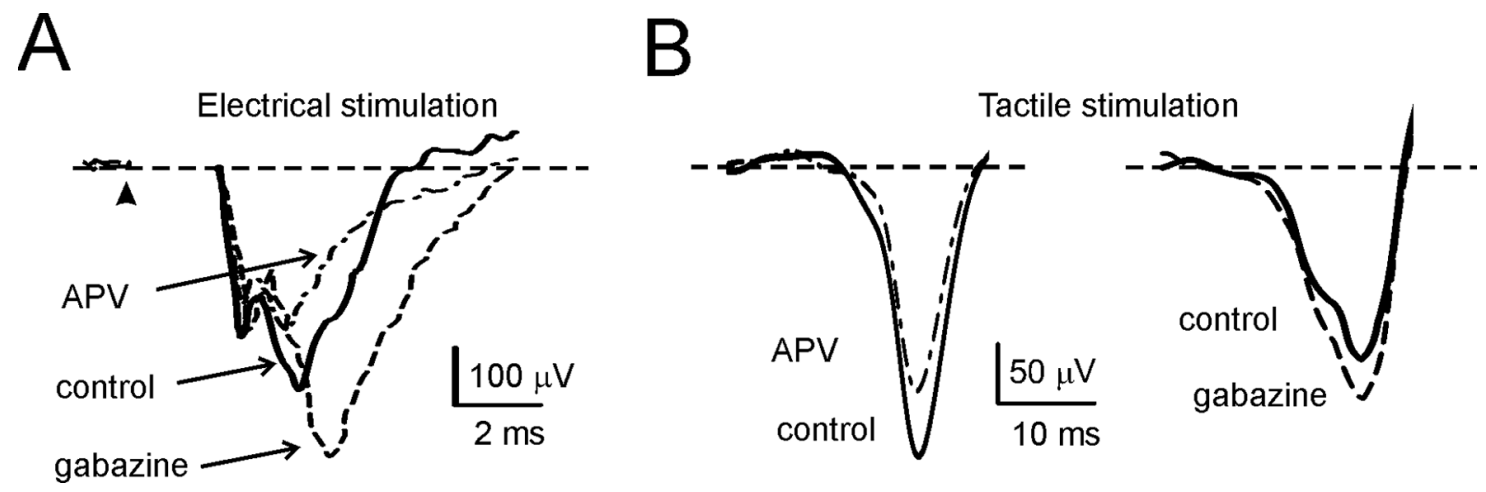

Figure 2. Pharmacological characterization of local field potentials. $A$, Pharmacological characterization of the $\mathrm{N}_{2}$ wave in response to intracerebellar electrical stimulation. The traces illustrate a sequential experiment in which, after a control period, drugs were applied on the cerebellar surface. The control wave (continuous trace) is increased by application of the $G_{A B A}$ receptor blocker gabazine (dashed trace). Subsequent application of the NMDA receptor blocker, D-APV (dashed-dotted trace), reduces the wave. Each trace is the average of 20 consecutive responses. $\boldsymbol{B}$, Pharmacological characterization of the T wave in response to tactile stimulation. The LFP is reported from two different experiments in which, after a control period (continuous trace), drugs were applied locally on the cerebellar surface. Application of D-APV (dashed-dotted trace) reduces, whereas application of gabazine (dashed trace) enhances, T. Each trace is the average of 20 consecutive responses.

logical classes (i.e., control vs gabazine vs gabazine plus APV) was tested using ANOVA (Press et al., 1992) (compare Figs. 3C and 4C).

\section{Results}

LFPs were recorded from the cerebellum granular layer in CrusIIa of anesthetized rats using either intracerebellar electrical stimulation or tactile stimulation of the whisker pad in two different experimental sets (Fig. 1 $A, B$ ). In both cases, the recording electrode was lowered until spontaneous activity of the molecular layer disappeared and evoked responses could be observed (Fig. $1 C)$. The response to tactile stimulation was broader and more complex than that to intracerebellar electrical stimulation, most probably reflecting the occurrence of spike bursts (rather than single spikes) in the mossy fibers and asynchronies in their activation (Vos et al., 1999; Chadderton et al., 2004) as well as the action of reverberant loops afferent to the cerebellum (Morissette and Bower, 1996; O'Connor et al., 2002).

The LFP generated by intracerebellar electrical pulses $(0.1 \mathrm{~Hz})$ resembled the $\mathrm{N}_{1}-\mathrm{N}_{2}-\mathrm{P}_{2}$ complex (Fig. $1 C$ ) originally reported by Eccles et al. (1967) using juxtafastigial stimulation in the cat. The response amplitude was measured at the peak of $\mathrm{N}_{2}$, which is mostly generated by granule cell excitation (Maffei et al., 2002; Mapelli and D'Angelo, 2007). $\mathrm{N}_{2}$ peaked in $5.4 \pm 0.9 \mathrm{~ms}(n=15)$ after the stimulus (corresponding therefore to $\mathrm{N}_{2 \mathrm{~b}}$ in slices) and was over in 2-3 ms.

The LFP generated by tactile stimuli $(0.1 \mathrm{~Hz})$ was composed of a characteristic sequence of three waves that were called T, C, and $\mathrm{D}$ (Fig. $1 B)$. T arose at $13.36 \pm 0.4 \mathrm{~ms}(n=15)$ and peaked at $17.9 \pm 0.6 \mathrm{~ms}(n=15) . \mathrm{C}$ arose at $25.7 \pm 0.9 \mathrm{~ms}(n=15)$ and peaked at $33.2 \pm 1.5 \mathrm{~ms}(n=15)$. The $\mathrm{D}$ wave was broader than $\mathrm{T}$ and $\mathrm{C}$, arose at $53.9 \pm 0.5 \mathrm{~ms}(n=15)$, and peaked at $112.8 \pm$ $3.0 \mathrm{~ms}(n=15)$. T and $\mathrm{C}$ corresponded to the trigeminal and cortical responses previously observed in Crus-IIa (Bower and Woolston, 1983; Morissette and Bower, 1996) (L. Roggeri and E. D'Angelo, unpublished observations). The D (delayed) wave was not reported previously [but see Lu et al. (2005), their Fig. 7] and may correspond to signals elaborated in brainstem circuits (Kleinfeld et al., 1999; O'Connor et al., 2002).

In the following investigation of granular layer responses to tactile stimuli, to obtain precise measurements of amplitude changes, only the $\mathrm{T}$ wave was considered (the analysis of $\mathrm{C}$ and $\mathrm{D}$ waves was hampered by their dependency on previous signal components and was not considered further). The $\mathrm{T}$ wave has also the advantage of establishing a direct link (via the trigeminal nucleus) between the sensory input and the cerebellum (Morissette and Bower, 1996), avoiding the potential effects of plasticity in the thalamocorticopontine and precerebellar circuits. Finally, the $\mathrm{T}$ wave could be directly compared with $\mathrm{N}_{2}$.

\section{Regulation of granular layer LFP by NMDA and GABA receptors}

The sensitivity of LFP to local excitatory and inhibitory connections was tested by applying specific receptor blockers (Fig. 2). It was of primary interest to test the role of NMDA receptors (Garthwaite and Brodbelt, 1990), which enhance transmission along the mossy fiber pathway (Kinney and Slater, 1992), promoting EPSP temporal summation and spike discharge in granule cells (D'Angelo et al., 1995), and determine the induction of mossy fiber-granule cell long-term synaptic plasticity (D'Angelo et al., 1999). Application of the competitive NMDA receptor antagonist APV $\left(500 \mu \mathrm{M}\right.$ on the cerebellar surface) reduced $\mathrm{N}_{2}$ $(-46.2 \pm 4.5 \% ; n=5 ; p<0.001$, paired $t$ test $)$ and $\mathrm{T}(-18.7 \pm$ $2.6 \% ; n=5 ; p<0.001$, paired $t$ test), indicating that responses were enhanced by NMDA receptor activation (Fig. $2 A$ ). NMDA channel voltage-dependent unblock, which is essential to regulate mossy fiber-granule cell neurotransmission and plasticity, is under strict control of the Golgi cell inhibitory circuit (Mapelli and D'Angelo, 2007). The role of inhibition in determining granular layer field responses was assessed by applying the $\mathrm{GABA}_{\mathrm{A}}$ receptor antagonist, gabazine (500 $\mu \mathrm{M}$ on the cerebellar surface). Application of gabazine enhanced $\mathrm{N}_{2}(+84.9 \pm 29.1 \% ; n=5$; $p<0.001$, paired $t$ test $)$ and $\mathrm{T}(+11.2 \pm 7.6 \% ; n=5 ; p<0.05$, paired $t$ test), indicating that the granular layer was under inhibitory control (Fig. 2A) (Chadderton et al., 2004). Thus, the two main regulatory mechanisms of granular layer responses were active in LFP evoked in vivo as well as in vitro. That the changes observed in $\mathrm{T}$ were smaller than those in $\mathrm{N}_{2}$ may reflect the different interaction of excitation and inhibition that is established in the local circuit when short busts rather than single impulses activate the circuit (S. Diwakar and E. D'Angelo, unpublished observations).

\section{Granular layer long-term synaptic plasticity in vivo}

To demonstrate whether the granular layer in vivo was capable of generating long-term synaptic plasticity, we have performed experiments using either electrical or tactile stimulation. 
A

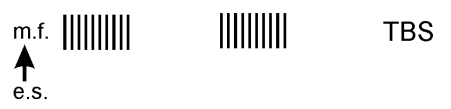

B

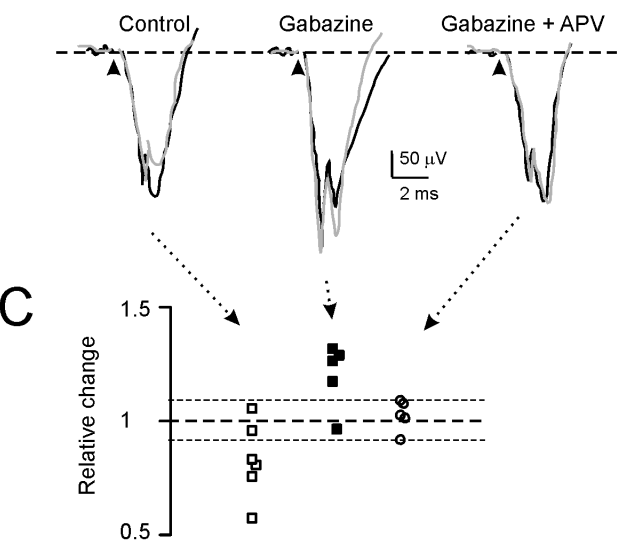

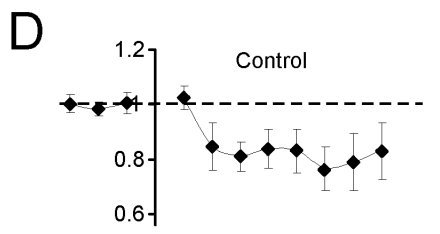
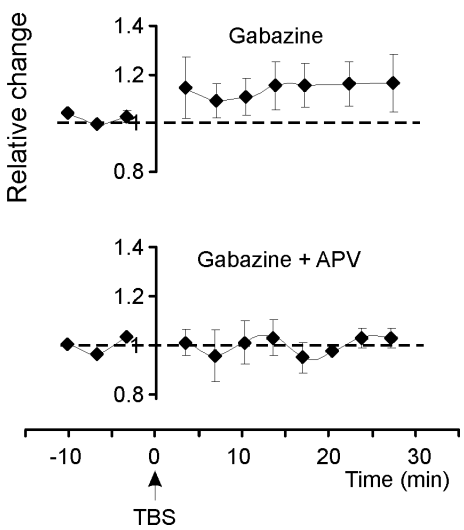

Figure 3. Long-term plasticity evoked by TBS in the N2 wave (intracerebellar electrical stimulation). $A$, The induction protoco (TBS) is shown. e.s., Electrical stimulation; m.f., mossy fiber. $\boldsymbol{B}$, Exemplar traces in different experimental conditions. Black traces are taken before TBS and gray traces 30 min after TBS. In control, $N_{2}$ is depressed; in the presence of gabazine, $N_{2}$ is enhanced; and in the presence of both gabazine and D-APV, $\mathrm{N}_{2}$ does not change. Each trace is the average of 20 consecutive responses. $\boldsymbol{C}$, The scatter plot shows $\mathrm{N}_{2}$ amplitude changes 30 min after TBS in individual experiments. Note the prevalence of experiments showing depression in control and LTP in the presence of gabazine, whereas no remarkable changes are observed in the presence of both gabazine and D-APV. The dotted lines represent $\pm 8 \%$ change. $D$, Time course of $\mathrm{N}_{2}$ amplitude changes for the same experiments shown in C. Top, In control, $\mathrm{N}_{2}$ shows LTD. Middle, After application of gabazine, $\mathrm{N}_{2}$ shows LTP. Bottom, After coapplying gabazine and D-APV, no significant $\mathrm{N}_{2}$ changes are observed. Data points are mean \pm SEM.

A

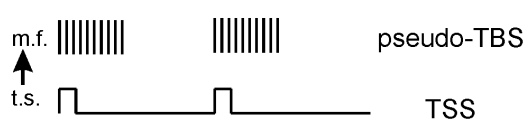

B

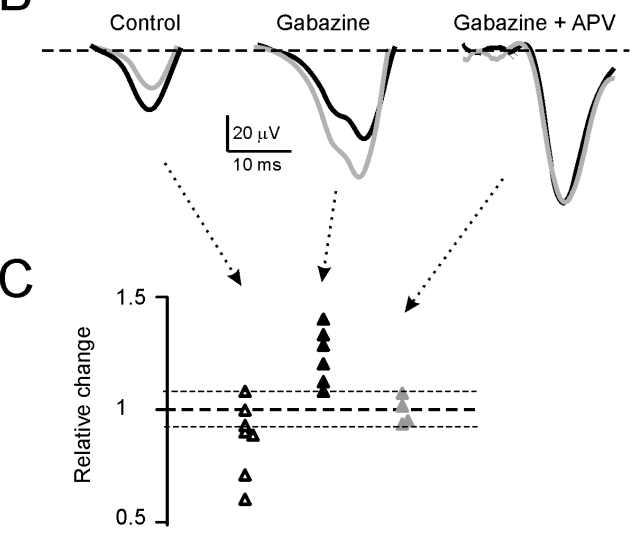

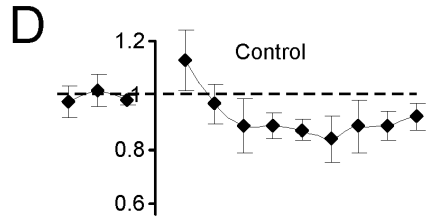
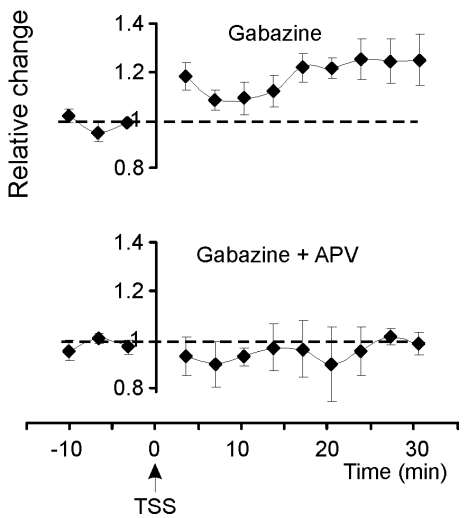

Figure 4. Long-term plasticity evoked by TSS in the T wave (tactile stimulation). $\boldsymbol{A}$, The induction protocol (TSS) and its expected consequence on mossy fiber discharge are shown. Note the transformation of a theta sensory stimulation into a pseudoTBS. t.s., Tactile stimulation; m.f., mossy fiber. $\boldsymbol{B}$, Exemplar traces in different experimental conditions. Black traces are taken before TSS and gray traces 30 min after TSS. In control, $\mathrm{T}$ is depressed; in the presence of gabazine, $\mathrm{T}$ is enhanced; and in the presence of both gabazine and D-APV, T does not change. Each trace is the average of 20 consecutive responses. $C$, The scatter plot shows T amplitude changes $30 \mathrm{~min}$ after TBS in individual experiments. Note the prevalence of experiments showing depression in control and LTP in the presence of gabazine, whereas no remarkable changes were observed in the presence of both gabazine and D-APV. The dotted lines represent $\pm 8 \%$ change. $D$, Time course of T amplitude changes for the same experiments shown in C. Top, In control, T shows LTD. Middle, After application of gabazine, T shows LTP. Bottom, After coapplying gabazine and D-APV, no significant $T$ changes are observed. Data points are mean \pm SEM.

Because a theta-burst stimulation (TBS; $10 \times 100 \mathrm{~Hz}$ bursts at $4 \mathrm{~Hz})$ pattern proved efficient in vitro to induce LTP and LTD (Armano et al., 2000; Rossi et al., 2002; Gall et al., 2005; Mapelli and D'Angelo, 2007), the same was applied through the intracerebellar electrode in experiments using electrical stimulation (Fig. 3A). A different strategy was used in experiments with tactile stimulation: because each air puff has been reported to elicit a short spike burst in the mossy fibers (Vos et al., 1999; Chadderton et al., 2004), we reasoned that TBS could be mimicked by applying the air puff at theta-frequency $(4 \mathrm{~Hz})$, configuring what we called theta-sensory stimulation (TSS). By analogy with TBS, a typical TSS was composed of 10 stimuli at 4 $\mathrm{Hz}$ (Fig. 4A). Using both electrical and tactile stimulations, different experiments were performed in control conditions (no added drugs), in the presence of gabazine, or in the presence of gabazine plus APV. These tests allowed a straightforward comparison with results previously reported in field recordings in vitro (Mapelli and D’Angelo, 2007).

The results of experiments performed using TBS and TSS are reported in Figures 3 and 4, respectively, and in Table 1 . The traces (Figs. $3 B, 4 B$ ) show exemplar $\mathrm{N}_{2}$ and $\mathrm{T}$ changes $30 \mathrm{~min}$ after TBS, and the scatter plots (Figs. 3C, 4C) summarize data from individual recordings. Using both TBS and TSS, recordings showed significant LTD in control conditions and significant LTP in the presence of gabazine, but no significant changes in the presence of gabazine plus APV. The pharmacological sensitivity and the time course and intensity of changes (Figs. 3D, 4D) were similar to those reported in vitro (cf. Mapelli and D'Angelo, 2007), and no significant differences were revealed between TSS and TBS in their efficacy in generating LTP and LTD. It should be noted that changes in gabazine plus APV recordings fell within $\pm 8 \%$, a limit similar to that reported for whole-cell and field recordings in slices and assumed to represent a range of variation in control recordings (D'Angelo et al., 1999; Mapelli and D'Angelo, 2007). The statistical significance of the datasets in Figures $3 C$ and $4 C$ was further assessed using multiple variance analysis on Figures $3 D$ and $4 D$, confirming the significant difference of LTP and LTD results compared with APV plus gabazine results $\left[\mathrm{N}_{2}, p(F)<0.01(\mathrm{df}=1,15) ; \mathrm{T}, p(F)<0.01\right.$ $(\mathrm{df}=1,16)]$.

\section{LTP and LTD regulate the delay of} granular layer response to tactile stimulation

A prediction from cellular neurophysiology is that, in response to mossy fiber 


\begin{tabular}{llll}
\hline & Control & Gabazine & Gabazine plus APV \\
\hline TBS ( $N_{2} \%$ changes $)$ & $-19.6 \pm 9.7 \%(n=6 ; p<0.01)$ & $18.4 \pm 7.5 \%(n=5 ; p<0.01)$ & $2.4 \pm 2.8 \%(n=5 ; p=0.78)$ \\
TSS (T \% changes) & $-12.3 \pm 6.5 \%(n=7 ; p<0.01)$ & $24.2 \pm 5.2 \%(n=6 ; p<0.01)$ & $-1.54 \pm 3.7 \%(n=4 ; p=0.32)$ \\
TBS versus TSS & $p=0.42$ & $p=0.74$ & $p=0.63$ \\
\hline
\end{tabular}

Data are reported $30 \mathrm{~min}$ after induction. Case-control statistics were done using paired Student's $t$ test. Comparisons between TBS and TSS were done using unpaired Student's $t$ test.

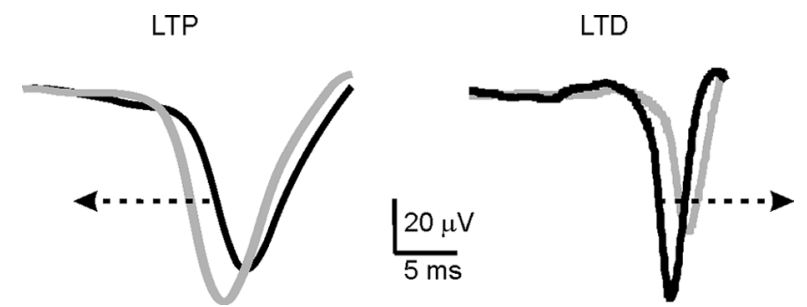

Figure 5. T waveform shifts after TSS. The traces show the average T wave obtained by pooling sweeps from experiments showing either $\operatorname{LTD}(n=5$ in control) or $\operatorname{LTP}(n=5$ in the presence of gabazine) $>8 \%$. The black traces are taken before TSS, and the gray traces are taken 30 min after TSS. It should be noted that T delay from the tactile stimulus is reduced during LTP and increased during LTD (arrows).

bursts, the delay of granule cell firing should decrease with LTP and increase with LTD (Nieus et al., 2006). Actually (Fig. 5), the T latency during LTD increased by $2.4 \pm 0.5 \mathrm{~ms}$ from control value ( $n=5 ; p<0.001$, paired $t$ test), whereas during LTP it decreased by $2.0 \pm 0.5 \mathrm{~ms}(n=5 ; p<0.003$, paired $t$ test $)$. This observation suggests that the mechanisms of mossy fiber LTP and LTD analyzed in acute cerebellar slices could determine response timing regulation in vivo.

\section{Discussion}

The main observation in this study is that tactile stimulation of the whisker pad can induce long-term synaptic plasticity in the field response recorded from the granular layer of cerebellum in the anesthetized rat. Given its induction pattern, pharmacology, and kinetics, granular layer plasticity in vivo was compatible with that observed in vitro at the mossy fiber-granule cell relay (Mapelli and D'Angelo, 2007). The similarity of LTP and LTD induced by tactile and intracerebellar electrical stimulation, by ruling out relevant changes occurring upstream along the transmission pathway (i.e., in the trigeminal nucleus), further indicated that the main site of plasticity had to reside in the granular layer. As predicted on the basis of the expression mechanisms of mossy fiber-granule cell synaptic plasticity, which involve a change in release probability capable of regulating the delay of granule cell discharge (Sola et al., 2004; Nieus et al., 2006), LTP anticipated and LTD delayed the T wave elicited by tactile stimulation over a range of $\sim 5 \mathrm{~ms}$.

The properties of $\mathrm{T}$ and $\mathrm{N}_{2}$ responses conformed to known principles of granular layer circuit physiology. The $\mathrm{T}$ was longer than $\mathrm{N}_{2}$ wave, probably because a short mossy fiber burst rather than a single spike was generated by punctate stimulation (Kase et al., 1980; Vos et al., 1999; Chadderton et al., 2004) (this interpretation is supported by unpublished mathematical simulations by S. Diwakar and E. D'Angelo). Actually, the sequence of tactile stimuli used to induce long-term synaptic plasticity effectively mimicked a theta-burst. The sensitivity of $\mathrm{T}$ and $\mathrm{N}_{2}$ to $\mathrm{GABA}_{\mathrm{A}}$ and NMDA receptor blockers was expected from recordings in vitro and was in agreement with the pharmacology of long-term synaptic plasticity (Maffei et al., 2002; Mapelli and D’Angelo, 2007). Thus, the prominence of LTD in control conditions could be explained by a limited activation of the NMDA receptor sys- tem under the strong inhibitory action exerted by Golgi cells, whereas LTP could be generated when inhibition was removed. The correspondence of pharmacological effects in vitro and in vivo indicated that urethane anesthesia did not critically impair the granular layer circuit functions under investigation.

The observation that long-term synaptic plasticity can be induced by tactile stimuli suggests that the cerebellum granular layer can be modified by activity in certain functional conditions. For instance, during whisking the sensorimotor system activates the cerebellum with a theta-frequency pattern (O'Connor et al., 2002; Szwed et al., 2003; Kleinfeld et al., 2006), which may be reinforced by recurrent loops endowed with appropriate time delays (Kistler and De Zeeuw, 2003). An important issue that remains open concerns how and when plasticity would be induced in freely behaving animals. It is possible that afferent signals would normally drive synapses into the LTD state, thus maintaining low granule cell excitability and sparse coding (Marr, 1969). However, signals presenting unusual combinations among receptive fields that prize excitation over inhibition [for the organization of excitatory and inhibitory receptive fields, see Vos et al. (1999) and Jörntell and Ekerot (2002)] may selectively lead certain groups of synapses into LTP (Mapelli and D'Angelo, 2007). Such a spatial organization may be strongly influenced by the anatomical connections of Golgi cell, which are activated by specific groups of mossy fibers and parallel fibers and inhibited by molecular layer interneurons [which, in turn, are activated by both parallel and climbing fibers (Barmack and Yakhnitsa, 2008)]. Moreover, gating mechanisms favoring the switch toward LTP might be provided by neuromodulators (like acetylcholine, serotonin, noradrenaline, or dopamine), which relate behavioral contexts (e.g., attention, reward, or fear) and reach the cerebellum through specific projections from the brainstem [for summary of concepts and modeling predictions, see Schweighofer et al. (2001)]. Another open issue concerns the functional significance of granular layer adaptation. Our recent investigations suggest that LTP and LTD could regulate the spatiotemporal patterns transmitted to Purkinje cells, determining whether or not certain granule cell groups will be able to emit spikes within appropriate time windows (Nieus et al., 2006; Mapelli and D'Angelo, 2007). This mechanism may serve to tune the granular layer response toward delays generated in the tactile afferent pathways (Johansson and Birznicks, 2004) and to adapt temporal correlations in the order of tens of milliseconds [e.g., in the eyeblink reflex (De Zeeuw and Yeo, 2005; Koekkoek et al., 2005)].

In conclusion, LTP and LTD in the granular layer, together with other forms of plasticity reported in the molecular layer (Jörntell and Ekerot, 2002; Gao et al., 2003) and in deep cerebellar nuclei (Ohyama et al., 2006) in vivo, have the potential of regulating the spatiotemporal patterns of activity along the mossy fiber pathway. The selective impact of mossy fiber-granule cell LTP and LTD on animal behavior remains largely to be determined through the experimental analysis of mice with neuronspecific mutations (Rossi et al., 2002; Koekkoek et al., 2005; Boyden et al., 2006) and the development of appropriate compu- 
tational models (Medina and Mauk, 2000; Schweighofer et al., 2001; Kistler and De Zeeuw, 2003).

\section{References}

Armano S, Rossi P, Taglietti V, D’Angelo E (2000) Long-term potentiation of intrinsic excitability at the mossy fiber-granule cell synapse of rat cerebellum. J Neurosci 20:5208-5216.

Barmack NH, Yakhnitsa V (2008) Functions of interneurons in mouse cerebellum. J Neurosci 28:1140-1152.

Bengtsson F, Jörntell H (2007) Ketamine and xylazine depress sensoryevoked parallel fiber and climbing fiber responses. J Neurophysiol 98:1697-1705.

Bliss TV, Collingridge GL (1993) A synaptic model of memory: long-term potentiation in the hippocampus. Nature 361:31-39.

Bliss TV, Collingridge GL, Morris RG (2003) Long-term potentiation and structure of the issue. Philos Trans R Soc Lond B Biol Sci 358:607-611.

Bower JM, Woolston DC (1983) Congruence of spatial organization of tactile projections to granule cell and Purkinje cell layers of cerebellar hemispheres of the albino rat: vertical organization of cerebellar cortex. J Neurophysiol 49:745-766.

Boyden ES, Katoh A, Pyle JL, Chatila TA, Tsien RW, Raymond JL (2006) Selective engagement of plasticity mechanisms for motor memory storage. Neuron 51:823-834.

Chadderton P, Margrie TW, Häusser M (2004) Integration of quanta in cerebellar granule cells during sensory processing. Nature 428:856-860.

D’Angelo E, De Filippi G, Rossi P, Taglietti V (1995) Synaptic excitation of individual rat cerebellar granule cells in situ: evidence for the role of NMDA receptors. J Physiol (Lond) 484:397-413.

D’Angelo E, Rossi P, Armano S, Taglietti V (1999) Evidence for NMDA and mGlu receptor-dependent long-term potentiation of mossy fiber-granule cell transmission in rat cerebellum. J Neurophysiol 81:277-287.

De Schutter E (1995) Cerebellar long-term depression might normalize excitation of Purkinje cells: a hypothesis. Trends Neurosci 18:291-295.

De Zeeuw CI, Yeo CH (2005) Time and tide in cerebellar memory formation. Curr Opin Neurobiol 15:667-674.

Eccles JC, Ito M, Szentagothai J (1967) The cerebellum as a neuronal machine. Berlin: Springer.

Franks NP, Lieb WR (1994) Molecular and cellular mechanisms of general anaesthesia. Nature 367:607-614.

Friedberg MH, Lee SM, Ebner FF (1999) Modulation of receptive field properties of thalamic somatosensory neurons by the depth of anesthesia. J Neurophysiol 81:2243-2252.

Gall D, Prestori F, Sola E, D’Errico A, Roussel C, Forti L, Rossi P, D’Angelo E (2005) Intracellular calcium regulation by burst discharge determines bidirectional long-term synaptic plasticity at the cerebellum input stage. J Neurosci 25:4813-4822.

Gao W, Dunbar RL, Chen G, Reinert KC, Oberdick J, Ebner TJ (2003) Optical imaging of long-term depression in the mouse cerebellar cortex in vivo. J Neurosci 23:1859-1866.

Garthwaite J, Brodbelt AR (1990) Glutamate as the principal mossy fibre transmitter in rat cerebellum: pharmacological evidence. Eur J Neurosci 2:177-180.

Hansel C, Linden DJ, D'Angelo E (2001) Beyond parallel fiber LTD: the diversity of synaptic and non-synaptic plasticity in the cerebellum. Nat Neurosci 4:467-475.

Hara K, Harris RA (2002) The anesthetic mechanisms of urethane: the effects on neurotransmitter-gated ion channels. Anesth Analg 94:313-318.

Ito M (1984) The cerebellum and neuronal control. New York: Raven.

Ito M (2006) Cerebellar circuitry as a neuronal machine. Prog Neurobiol 78:272-303.

Ito M, Sakurai M, Tongroach P (1982) Climbing fibre induced depression of both mossy fibre responsiveness and glutamate sensitivity of cerebellar Purkinje cells. J Physiol (Lond) 324:113-134.

Johansson R, Birznicks I (2004) First spikes in ensembles of human tactile afferents code complex spatial fingertip events. Nat Neurosci 7:170-177.

Jörntell H, Ekerot CF (2002) Reciprocal bidirectional plasticity of parallel fiber receptive fields in cerebellar Purkinje cells and their afferent interneurons. Neuron 34:797-806.

Jörntell H, Ekerot CF (2006) Properties of somatosensory synaptic integration in cerebellar granule cells in vivo. J Neurosci 26:11786-11797.

Jörntell H, Hansel C (2006) Synaptic memories upside down: bidirectional plasticity at cerebellar parallel fiber-Purkinje cell synapses. Neuron 52:227-238.

Kase M, Miller DC, Noda H (1980) Discharges of Purkinje cells and mossy fibres in the cerebellar vermis of the monkey during saccadic eye movements and fixation. J Physiol (Lond) 300:539-555.

Kinney GA, Slater NT (1992) Potentiation of mossy fiber-evoked EPSPs in turtle cerebellar Purkinje cells by the metabotropic glutamate receptor agonist 1S,3R-ACPD. J Neurophysiol 67:1006-1008.

Kistler WM, De Zeeuw C (2003) Time windows and reverberating loops: a reverse engineering approach to cerebellar function. Cerebellum 2:44-54.

Kleinfeld D, Berg RW, O'Connor SM (1999) Anatomical loops and their electrical dynamics in relation to whisking by rat. Somatosens Mot Res 16:69-88.

Kleinfeld D, Ahissar E, Diamond ME (2006) Active sensation: insights from the rodent vibrissa sensorimotor system. Curr Opin Neurobiol 16:435-444.

Koekkoek SKE, Yamaguchi K, Milojkovic BA, Dortland BR, Ruigrok TJH, Maex R, Graaf WD, Smit AE, VanderWerf F, Bakker CE, Willemsen R, Ikeda T, Kakizawa S, Onodera K, Nelson DL, Mientjes E, Joosten M, De Schutter E, Oostra BA, Ito M, et al. (2005) Deletion of FMR1 in Purkinje cells enhances parallel fiber LTD, enlarges spines, and attenuates cerebellar eyelid conditioning in Fragile X syndrome. Neuron 47:339-352.

Lu H, Hartmann MJ, Bower JM (2005) Correlations between Purkinje cell single-unit activity and simultaneously recorded field potentials in the immediately underlying granule cell layer. J Neurophysiol 94:1849-1860.

Maffei A, Prestori F, Rossi P, Taglietti V, D’Angelo E (2002) Presynaptic current changes at the mossy fiber-granule cell synapse of cerebellum during LTP. J Neurophysiol 88:627-638.

Malenka RC, Nicoll RA (1999) Long-term potentiation-a decade of progress? Science 285:1870-1874.

Mapelli J, D'Angelo E (2007) The spatial organization of long-term synaptic plasticity at the input stage of cerebellum. J Neurosci 27:1285-1296.

Marr DA (1969) A theory of cerebellar cortex. J Physiol (Lond) 202:437-470.

Medina JF, Mauk MD (2000) Computer simulation of cerebellar information processing. Nat Neurosci 3 [Suppl]:1205-1211.

Morissette J, Bower JM (1996) Contribution of somatosensory cortex to responses in the rat cerebellar granule cell layer following peripheral tactile stimulation. Exp Brain Res 109:240-250.

Nieus T, Sola E, Mapelli J, Saftenku E, Rossi P, D’Angelo E (2006) LTP regulates burst initiation and frequency at mossy fiber-granule cell synapses of rat cerebellum: experimental observations and theoretical predictions. J Neurophysiol 95:686-699.

O'Connor SM, Berg RW, Kleinfeld D (2002) Coherent electrical activity between vibrissa sensory areas of cerebellum and neocortex is enhanced during free whisking. J Neurophysiol 87:2137-2148.

Ohyama T, Nores WL, Medina JF, Riusech FA, Mauk MD (2006) Learninginduced plasticity in deep cerebellar nucleus. J Neurosci 26:12656-12663.

Press WH, Teukilsky SA, Vetterling WT, Flannery BP (1992) Numerical recipes. Cambridge, UK: Cambridge UP.

Rossi P, Sola E, Taglietti V, Borchardt T, Steigerwald F, Utvik K, Ottersen OP, Kohr G, D'Angelo E (2002) Cerebellar synaptic excitation and plasticity require proper NMDA receptor positioning and density in granule cells. J Neurosci 22:9687-9697.

Schweighofer N, Doya K, Lay F (2001) Unsupervised learning of granule cell sparse codes enhances cerebellar adaptive control. Neuroscience 103:35-50.

Shambes GM, Gibson JM, Welker W (1978) Fractured somatotopy in granule cell tactile areas of rat cerebellar hemispheres revealed by micromapping. Brain Behav Evol 15:94-140.

Sola E, Prestori F, Rossi P, Taglietti V, D’Angelo E (2004) Increased neurotransmitter release during Long-term Potentiation at mossy fibre-granule cell synapses in rat cerebellum. J Physiol (Lond) 557:843-861.

Szwed M, Bagdasarian K, Ahissar E (2003) Encoding of vibrissal active touch. Neuron 40:621-630.

Vos BP, Volny-Luraghi A, De Schutter E (1999) Cerebellar Golgi cells in the rat: receptive fields and timing of responses to facial stimulation. Eur J Neurosci 11:2621-2634.

Welsh JP, Yamaguchi H, Zeng X, Kojo M, Nakada Y, Takagi A, Sugimori M, Llinás RR (2005) Normal motor learning during pharmacological prevention of Purkinje cell long-term depression. Proc Natl Acad Sci USA 102:17166-17171. 